\title{
Response uncertainty and perceptual difficulty of auditory temporal patterns'
}

\author{
FRED L. ROYER ${ }^{2}$, VETERANS ADMINISTRATION HOSPITAL, PERRY POINT, MARYLAND
}

W. R. GARNER, JOHNS HOPKINS UNIVERSITY

Two qualitatively different sounds were used to generate 256 different sequences of length 8 , and these sequences were presented to $S$ s at a rate of two stimuli per second. These sequences, when repeated continuously, can be grouped into 20 fundamentally different patterns, each having either 2,4 , or 8 distinguishably different starting points. Ss were required to listen and to begin responding (with telegraph keys) in synchrony to the patterns when they were able. The point at which they began responding, the delay before responding, and errors after beginning responding were measured. The response uncertainty (variability of point of response for a given pattern), average delay, and average errors are all highly correlated, indicating that pattems which are easily organized are those which have few alternative modes of organization, and thus can be considered as simple, or good in the Gestalt sense.

The psychological literature on pattern perception deals almost exclusively with visual pattern. (E.g., see bibliographic citations in Day, 1964.) While there has been relatively little direct experimentation on auditory pattern perception, it is not unreasonable to assume that principles of perceptual organization which apply to the visual modality also apply to the auditory modality. In applying these principles of visual pattern perception to audition, however, it is almost certainly necessary to restate the nature of the patterns in terms of time rather than of space. While auditory patterns of frequency, for example, can exist, temporal patterns are far more common and probably fundamental to the nature of the auditory process.

Visual patterns can exsist in space, be describable with respect to the space their elements occupy, and yet not be patterns emerging out of variations of spatial dimensions. To illustrate, it is possible to arrange five dots so that they form a cross. Such a cross is a pattern in space but not of space, since within broad limits the distances between the dots may be changed without affecting the pattern itself.

Analogously, auditory patterns can exist in time but not be of time. For example, rhythmic pattern in music may be produced by melody, harmony, or instrumentation, or as Meyer $(1956, p .103)$ puts it, by ".. . any other mode of articulation which can differentiate one stimulus or group of stimuli from others. Even a silence, a rest, may be accented..." Duration of an auditory pattern element may itself be a variation in time rather than of time, since if each successive element begins at a fixed time interval from the beginning of the last element, there is no time variation between element locations. Woodrow (1909), in his classic study on duple and triple feet, demonstrated that differences in either duration or intensity of elements affected the perceived temporal interval between successive events, i.e., determined the grouping of events into patterns.

Since the perceived pattern, as opposed to the objectively presented pattern, involves variations in apparent intensity and duration of elements as well as the interval between elements (see Farnsworth, 1958 , p. 70), use of these physical parameters to produce auditory pattern is probably less satisfactory than the use of elements which are qualitatively different but which have equal intensity and duration.

The problem of complexity or, inversely, "goodness" of unique patterns has been considered by Garner (1962). He suggested that simple or good patterns are perceived by the observer as coming from smaller inferred subsets of patterns than do the complex patterns, and thus as having higher psychological redundancy (or lower uncertainty) than complex patterns. Garner and Clement (1963) tested this suggestion with visual patterns generated by placing dots in the cells of an imaginary $3 \times 3$ matrix. They found that the judged goodness of the patterns was inversely correlated with the size of the subsets formed when figures were sorted according to perceived equivalence. In addition to this behavioral measure of the size of subset, they found that subset size differences produced by the objective criteria of rotation and reflection of patterns were also highly related to perceived goodness of pattern.

The simplest way to generate auditory temporal patterns is to use dichotomous elements, qualitatively different, in sequences of specified length which repeat themselves exactly. The number of such patterns which can be produced depends on the length of the sequence, and increases geometrically with the length. For any length, however, these sequences, when repeated in definitely, organize themsleves into groups of equivalent sequences. For example, the sequence 001 and 010 are identical with continued repetition. For any specified length there will be a number of mutually exclusive subsets of such sequences which will include all possible sequences, and these subsets will differ in size, i.e., the number of equivalent sequences. Thus we have an objective measure of subset size comparable to the rotation and reflection criteria used by Garner and Clement. 
In addition, however, just as with the visual patterns, there can be differences in subjective, or psychologically inferred, subset size which can be related to goodness of auditory pattern.

The purpose of this experiment was to investigate auditory pattern perception with sequences of dichotomous stimulus elements. While the experiment was designed to provide broad information about the nature of auditory temporal pattern perception, it was specifically designed to obtain a measure of psychological uncertainty of sequences of fixed length and also measures of difficulty of perceptual organization of the patterns, in the expectation that these measures would be correlated.

\section{Stimuli \\ METHOD}

Source. The auditory stimuli were produced by two annunciator buzzers mounted to produce sounds of approximately the same intensity differing noticeably in quality. These stimuli constituted the elements of the sequences.

All of the 256 different binary sequences having a length of eight units were used. These sequences were programmed by a parallel-input, serial-output, recirculating 8-bit shift register. The stimuli were presented at a rate of $2 / \mathrm{sec}$. with a constant duration of approximately $0.25 \mathrm{sec}$.

Patterns. Equivalences among the 256 different sequences exist by reason of complementarity and cyclic variation. These equivalences allow the formation of 20 basically different patterns. Thus, sequences $11111110,11111101,11111011$, etc. are cyclic variations of one pattern. One such sequence is, arbitrarily, the referent pattern and is indicated in Table 1.

Starting Points. Each sequence within each pattern is identifiable by the position of its starting point (SP) in the referent pattern. For example, the referent of pattern $E$ is 11111110 ; sequence 11110111 is therefore pattern $E$ at the 4 th SP. The number of different SPs for each pattern is shown in Table 1. Three patterns have less than eight distinguishably different SPs; these patterns are expansions, through repetition, of patterns of shorter unit-length.

Complements. Each sequence has a complementary form (CF). Complements were subsumed under the referent pattern and where necessary are identified with a prime symbol. Thus, sequence 90000100 is pattern $E^{\prime}$ at the 3rd SP.

Four patterns are internally complementary; that is, for any given SP, the sequence defined is the complement of another sequence defined by a different SP. For example, eight of the 256 sequences are classifed as pattern $F$. The sequence 00001111 , pattern $F$ at the 4th SP, is the complement of pattern $F$ at the 1st SP. All such sequences were considered to have only one complementary form.
The number of complementary forms for each of the SPs is shown in Table 1. Thus the total number of sequences for each pattern is determined by the number of SPs times the number of complements for each SP.

\section{Procedure}

Subjects. The Ss were 32 men from the Bainbridge Naval Training Center who were awaiting the convocation of a class at the Radio School. Their ages ranged from 17 to 21 years.

Experimental Design. The maximum testing time per subject was set at 1-3/4 hours with 20 minutes for rest time. This period was convenient to military schedules and, on the basis of pilot work, was found not to be overly taxing of S's motivation.

The Ss were divided into four groups, each group being presented with one of four lists of sequences containing 64 of the 256 different sequences. The first division of Ss was made according to complementarity of patterns. The complements of each different sequence in a pattern were distributed between two lists so as to control for the frequency of occurrence of the 2 sounds at the beginning of the sequence.

The second division of Ss was dictated by the limitations of the testing time. All of those patterns formed from less than 16 different sequences were assigned to one division. The remaining patterns were distributed between the two divisions.

As finally composed, Lists $A$ and $A$ ' consisted of all SPs and complements of patterns A, B, C, D, F, G, H, $I, M, P, S$, and $T$. Lists $B$ and $B^{\prime}$ contained, in similar fashion, patterns $E, J, K, L, N, O, Q$, and $R$.

$S$ received one SP for each of his patterns before a different SP of the same pattern was used. A latin square arrangement was used to counter-balanceorder of pattern across Ss, and a second latin square (orthogonal to the first) was used to counterbalance SP within pattern across $\mathrm{Ss}$. This was made possible by treating patterns $A, B, C$, and $S$ and patterns $D$ and $F$ as single columns of the matrix for lists $A$ and $A '$.

Task. $\mathrm{S}$ was seated before two telegraph keys mounted about 2-1/2 inches apart. The buzzers were placed to the right and left of S. He was instructed to press the keys to learn which tone was associated with which key. The buzzers were then detached from the keys and placed under the control of the automatic programmer. $S$ was then instructed:

I have now connected the buzzers so that they will be sounded automatically by this apparatus. When the apparatus is turned on, the buzzers will sound at regular intervals, like a pulse. There will be a regular beat (demonstrate with hand hitting table), without pauses, continuing until I turn it off. The buzzer will sound in some kind of pattern. This will continue until I turn it off. I would like you to listen and when you feel you can follow the series of sounds, press the keys corresponding to the appropriate buzzer. That is, for a sound on the left press the left key; for a 
sound on the right, press the right key. If you have difficulty or make errors, feel free to stop and listen and begin again when you are ready. In summary, the buzzer will sound in some pattern. You will listen; when you feel you can follow the pattern, begin pressthe keys. You will be duplicating the pattern while the buzzers are sounding. Continue to press the keys, duplicating the pattern until I turn off the buzzers.

$\mathrm{S}$ was then given a series of eight practice patterns drawn from another list not composed of complements of the test 1ist; if tested on list A or $A^{\prime}$, he practiced on patterns from list $B$ or $B^{\prime}$ and vise versa. At the completion of the practice period, $S$ was given a rest of 10 minutes. Midway through the test list another 10 minute rest was given. Both stimuli and responses were recorded on an Esterline-Angus Event Recorder.

Criterion. A particular pattern was terminated by E when Shad correctly followed 16 consecutive elements. E observed the Esterline-Angus Recorder to determine when the criterion had been reached.

Point of Response. (a) Location. S was free to choose the point at which to begin his responding. The location of this point for response sequences culminating in the criterion was determined and identified by its equivalent point in the referent pattern. The frequency of the use of a particular point of response (RP) was tabulated for each pattern. Thus, in Table 1, there is a score for each possible distinguishably different point at which $\mathrm{S}$ could begin responding. This score is the frequency with which the point was used as the RP across all different SPs of the pattern.

(b) Response Incertainty. The uncertainty of the distribution of the RPs in bits was used as a summary measure of the variability of RPs.

Delay. The number of stimulus elements which occurred between the presentation of the sequence and the point at which the S began a series of responses which culminated in achieving the criterion constitutes this score. As may be seen in Table 1, these scores are related to the SP of the sequence in the referent pattern.

Errors. This measure was the number of positive errors (non-synchronous responses) made prior to reaching criterion but made after the delay, and in Table 1 is tabulated with respect to the SP of the presented sequence in the referent pattern. Other measures of error such as omissions after an aborted response sequence or the total number of omissions and the positive errors, correlated with the number of positive errors greater than .92; these data are not presented.

\section{RESULTS}

\section{Point of Response}

Predominant Locations. The specific number of different starting positions and complementary forms of each pattern are shown in Table $1 .{ }^{3}$ Since the pattern was presented to eight $S s$ in each of its starting positions and complementary forms, the total number of responses for a particular pattern is the product of these values. These responses can je distributed across the same number of alternative points as there are SPs. In Table 1 the frequencies with which each of these points was used as RPare shown.

Since all SPs were used equally often in the presentation of a sequence, and since these SPs were counterbalanced across Ss and time, there is no objective factor in the experiment which would predispose some points in the sequence to be used as RPs more frequently than others. The fact that some points are used much more frequently than others must therefore reflect the perceived properties of the sequences-the reorganized pattern. Furthermore, the points in the sequence which are frequently used (the modes) must define the perceived beginnings of the reorganized patterns. In musical terms, these modes must correspond to accent points.

While there appear to be some idiosyncratic factors in the location of these modes, there are two general factors which emerge in determining this location: (1) Runs. A commonly used mode is the beginning of a run of three or more identical elements, as in pattern $\mathrm{J}$. Beginnings of shorter runs will also be used, however, when there are only two runs in the sequence as in patterns $\mathrm{E}$ and $\mathrm{H}_{\circ}(2)$ Single alternations. The beginning of at least two cycles of a single alternation commonly serves as an accent point except when this beginning is the end of a run of identical elements. For example, pattern $I$ is frequently begun as 01010111, but rarely as 10101011.

When neither of these conditions is met in a sequence, the patterns are very difficult and have high uncertainty, as in patterns $\mathrm{S}$ and $\mathrm{T}$.

Response Uncertainty. The uncertainty of the distribution of the RPs for all Ss for each pattern is shown in Table 1 as the summary statistic for the frequency distribution. This measure of the variability of the RP ranged from 0.99 to 2.72 over all patterns, and from 1.32 to 2.72 for patterns having eight SPs. In the latter case, there is a range of 1.40 bits of uncertainty in the response, which is the equivalent of a ratio of nearly 3 .

The ordering of the patterns in Table 1 is according to the response uncertainty. It is quite obvious from inspection that this measure is highly correlated with the complexity of the pattern. For this reason, direct judgments of complexity were considered superfluous and were not obtained.

In Gestalt psychology, the variations of forms from simple to complex are related to the degree of articulation which is itself a consequence of the integration of elements according to the principles of similarity and adjacency. We have seen that the location of the RPs is determined by runs of similar elements and by 
Table 1. Summary data for auditory binary sequential patterns of 8 -unit length.

\begin{tabular}{|c|c|c|c|c|c|c|c|c|c|c|}
\hline \multicolumn{9}{|c|}{$\begin{array}{l}\text { Pattern and response scores for equivalent } \\
\text { response points or starting points }\end{array}$} & \multirow[t]{2}{*}{$\begin{array}{c}\text { Summary } \\
\text { Statistics } 1\end{array}$} & \multirow{2}{*}{$\begin{array}{c}\text { N of SPs } \\
\& \text { CFs } \\
2 \times 1\end{array}$} \\
\hline B Pattern & 1 & $\mathbf{0}$ & $\mathbf{1}$ & 0 & 1 & $\mathbf{0}$ & 1 & 0 & & \\
\hline Freq. of RP & 7 & 9 & & & & & & & 0.99 & \\
\hline Median Delay & 11 & 12 & & & & & & & 11.5 & \\
\hline Total Errors & 3 & 0 & & & & & & & 0.19 & \\
\hline C Pattern & 1 & 1 & 0 & 0 & 1 & $\mathbf{1}$ & $\mathbf{0}$ & 0 & . & $4 \times 1$ \\
\hline Freq. of RP & 16 & 2 & 14 & 0 & & & & & 1.27 & \\
\hline Median Delay & 11 & 15 & 11 & 15 & & & & & 13.0 & \\
\hline Total Errors & 0 & 3 & 0 & 1 & & & & & 0.13 & \\
\hline
\end{tabular}

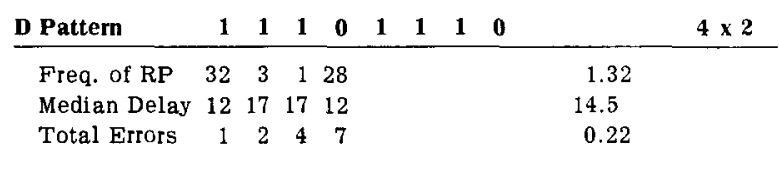

\begin{tabular}{|c|c|c|c|c|c|c|c|c|c|c|}
\hline E Pattern & 1 & 1 & 1 & 1 & 1 & 1 & 1 & 0 & & $8 \times 2$ \\
\hline Freq. of RP & 69 & 1 & 1 & 2 & 1 & 1 & 0 & 53 & 1.32 & \\
\hline Median Delay & 16 & 23 & 22 & 37 & 20 & 23 & 18 & 16 & 21.9 & \\
\hline Total Errors & 1 & 6 & 5 & 6 & 2 & 0 & 1 & 3 & 0.19 & \\
\hline F Pattem & 1 & 1 & 1 & 1 & 0 & $\mathbf{0}$ & $\mathbf{0}$ & 0 & & $8 \times 1$ \\
\hline Freq. of RP & 33 & 0 & 0 & 1 & 26 & 3 & 1 & 0 & 1.42 & \\
\hline Median Delay & 12 & 23 & 22 & 19 & 12 & 17 & 22 & 17 & 18.0 & \\
\hline Total Errors & 0 & 1 & 1 & 0 & 0 & 2 & 6 & 0 & 0.16 & \\
\hline G Pattern & 1 & 1 & 1 & 1 & 1 & $\mathbf{0}$ & $\mathbf{0}$ & 0 & & $8 \times 2$ \\
\hline Freq. of RP & 69 & 5 & 0 & 1 & 1 & 49 & 2 & 1 & 1.45 & \\
\hline Median Delay & 16 & 23 & 22 & 22 & 20 & 16 & 23 & 22 & 20.5 & \\
\hline Total Errors & 0 & 6 & 16 & 6 & 3 & 2 & 5 & 6 & 0.34 & \\
\hline H Pattern & 1 & 1 & $\mathbf{1}$ & $\mathbf{1}$ & 1 & 1 & 0 & $\mathbf{0}$ & & $8 \times 2$ \\
\hline Freq. of RP & 72 & 6 & 6 & 0 & 2 & 3 & 38 & 1 & 1.68 & \\
\hline Median Delay & 16 & 23 & 22 & 28 & 20 & 19 & 16 & 23 & 20.9 & \\
\hline Total Errors & 5 & 7 & 8 & 6 & 6 & 2 & 4 & 7 & 0.35 & \\
\hline
\end{tabular}

\begin{tabular}{|c|c|c|c|c|c|c|c|c|c|c|}
\hline I Pattem & 1 & 1. & 1 & $\mathbf{0}$ & 1 & 0 & 1 & $\mathbf{0}$ & & $8 \times 2$ \\
\hline Freq. of RP & 37 & 1 & 3 & 63 & 18 & 3 & 1 & 2 & 1.88 & \\
\hline Median Delay & 22 & 25 & 20 & 16 & 23 & 18 & 24 & 23 & 21.4 & \\
\hline Total Errors & 4 & 6 & 13 & 7 & 2 & 9 & 3 & 8 & 0.41 & \\
\hline J Pattern & 1 & 1 & 1 & $\mathbf{0}$ & 1 & $\mathbf{0}$ & $\mathbf{0}$ & 0 & & $8 \times 2$ \\
\hline Freq. of RP & 65 & 1 & 0 & 32 & 4 & 18 & 7 & 1 & 1.89 & \\
\hline Median Delay & 26 & 33 & 30 & 31 & 60 & 24 & 42 & 33 & 34.9 & \\
\hline Total Errors 1 & 109 & 47 & 33 & 20 & -219 & 22 & 44 & 24 & 4.05 & \\
\hline K Pattern & 1 & 1 & 1 & 1 & $\mathbf{0}$ & 0 & 1 & $\mathbf{0}$ & & $8 \times 2$ \\
\hline Freq. of RP & 36 & 5 & 3 & 2 & 61 & 3 & 16 & 2 & 2.02 & \\
\hline Median Delay & 32 & 37 & 28 & 41 & 24 & 44 & 32 & 29 & 33.4 & \\
\hline Total Errors & 20 & 23 & 34 & 24 & 18 & 34 & 41 & 10 & 1.59 & \\
\hline L Pattern & 1 & $\mathbf{1}$ & 1 & 1 & 1 & $\mathbf{0}$ & 1 & $\mathbf{0}$ & & $8 \times 2$ \\
\hline Freq. of RP & 49 & 4 & 1 & 2 & 8 & 50 & 11 & 3 & 2.05 & \\
\hline Median Delay & 16 & 26 & 29 & 26 & 23 & 30 & 24 & 22 & 24.5 & \\
\hline Total Errors & 3 & 9 & 10 & 10 & 5 & 7 & 17 & 9 & 0.55 & \\
\hline M Pattern & 1 & 1 & 1 & 1 & 0 & 1 & 0 & $\mathbf{0}$ & & $8 \times 2$ \\
\hline Freq. of RP & 54 & 6 & 3 & 2 & 45 & 3 & 11 & 4 & 2.07 & \\
\hline Median Delay & 16 & 24 & 30 & 29 & 24 & 35 & 36 & 21 & 26.9 & \\
\hline Total Errors & 11 & 19 & 14 & 10 & 12 & 14 & 39 & 6 & 0.98 & \\
\hline
\end{tabular}

alternations which may be thought of as a regular, temporally symmetrical arrangement of elements. It is clear that the variability of the distribution of the responses, i.e., the response uncertainty, is also correlated with the articulation of the sequences. Those patterns which consists of essentially two runs of similar elements, patterns $\mathrm{E}, \mathrm{F}, \mathrm{G}$, and $\mathrm{H}$ have relatively low response uncertainty. If we accept alternation as a perceptual unit, it will be seen that even pattern I is essentially made up of two runs. As the elements are more scattered and more irregular through the sequence, the response uncertainty increases.

The response uncertainty provides an index of the complexity of the sequence here just as it provided an index of the complexity of visual figures in the study by Garner and Clement (1963) where the response uncertainty described the size of the inferred subset of figures from which a particular figure was drawn. Consistencies of response of different Ss across SPS clearly demonstrates that the psychological alternatives are less than those logically possible. The variations in the degree of consistency, measured by the response uncertainty, reflect the number of alternatives. In this

\begin{tabular}{lrrrrrrrrrr} 
N Pattern & $\mathbf{1}$ & $\mathbf{1}$ & $\mathbf{1}$ & $\mathbf{1}$ & $\mathbf{0}$ & $\mathbf{1}$ & $\mathbf{1}$ & $\mathbf{0}$ & & $\mathbf{8} \times \mathbf{2}$ \\
\hline Freq. of RP & 40 & 1 & 2 & 0 & 45 & 26 & 4 & 10 & 2.11 & \\
Median Delay & 17 & 27 & 28 & 25 & 16 & 22 & 25 & 33 & 24.1 & \\
Total Errors & 7 & 2 & 8 & 7 & 7 & 17 & 7 & 32 & 0.58 & \\
& & & & & & & & & & \\
o Pattern & $\mathbf{1}$ & $\mathbf{1}$ & $\mathbf{1}$ & $\mathbf{0}$ & $\mathbf{1}$ & $\mathbf{1}$ & $\mathbf{0}$ & $\mathbf{0}$ & & $\mathbf{8} \times \mathbf{2}$ \\
\hline Freq. of RP & 61 & $\mathbf{3}$ & 1 & 31 & 11 & 3 & 14 & $\mathbf{4}$ & 2.12 & \\
Median Delay & 26 & 31 & 42 & $\mathbf{5 3}$ & 26 & 39 & 48 & 35 & 37.5 & \\
Total Errors & 117 & 20 & 33 & $\mathbf{3 4}$ & 15 & 63 & 40 & 20 & 2.67 &
\end{tabular}

\begin{tabular}{lrrrrrrrrrr} 
P Pattern & $\mathbf{1}$ & $\mathbf{1}$ & $\mathbf{1}$ & $\mathbf{0}$ & $\mathbf{0}$ & $\mathbf{1}$ & $\mathbf{1}$ & $\mathbf{0}$ & & $\mathbf{8} \times \mathbf{2}$ \\
\hline Freq. of RP & 21 & 2 & 2 & 52 & 2 & 38 & 2 & 9 & 2.12 & \\
Median Delay & 37 & 61 & 62 & 41 & 47 & 33 & 36 & 39 & 44.5 & \\
Total Errors & 30 & 61 & 26 & 39 & 54 & 38 & 32 & 29 & 2.41 &
\end{tabular}

\begin{tabular}{lrrrrrrrrrr} 
Q Pattern & 1 & $\mathbf{1}$ & $\mathbf{1}$ & $\mathbf{0}$ & $\mathbf{0}$ & $\mathbf{1}$ & $\mathbf{0}$ & $\mathbf{0}$ & & $\mathbf{8} \times \mathbf{2}$ \\
\hline Freq. of RP & 35 & 4 & 2 & 58 & 4 & 10 & 5 & 4 & 2.15 & \\
Median Delay & 27 & 33 & 29 & 28 & 42 & 37 & 29 & 41 & 33.0 & \\
Total Errors & 33 & 34 & 19 & 15 & 27 & 34111 & 25 & 2.33 &
\end{tabular}

\begin{tabular}{|c|c|c|c|c|c|c|c|c|c|c|}
\hline R Pattern & 1 & 1 & 0 & 1 & 0 & 1 & 0 & 0 & & $8 \times 2$ \\
\hline Freq. of RP & 40 & 4 & 53 & 6 & 4 & 3 & 15 & 3 & 2.19 & \\
\hline Median Delay & 32 & 29 & 24 & 32 & 24 & 39 & 32 & 32 & 30.5 & \\
\hline Total Errors & 10 & 32 & 16 & 11 & 10 & 36 & 14 & 43 & 1.34 & \\
\hline
\end{tabular}

\begin{tabular}{|c|c|c|c|c|c|c|c|c|c|}
\hline S Pattern & 1 & 1 & $\mathbf{0}$ & 1 & 0 & $\mathbf{0}$ & 1 & & $8 \times 1$ \\
\hline Freq. of RP & 14 & 0 & 6 & 51 & 11 & 122 & $22 \quad 5$ & 2.43 & \\
\hline Median Delay 1 & 190 & 74 & 5216 & 677 & 76 & 88112 & 1272 & 103.9 & \\
\hline Total Errors & 59 & 79 & $27 \quad 75$ & 792 & 27 & 3751 & 5123 & 5.97 & \\
\hline
\end{tabular}

\begin{tabular}{|c|c|c|c|c|c|c|c|c|c|c|}
\hline T Pattern & 1 & $\mathbf{1}$ & $\mathbf{0}$ & 1 & 1 & $\mathbf{0}$ & 1 & $\mathbf{0}$ & & $8 \times 2$ \\
\hline Freq. of RP & 29 & 4 & 21 & 16 & 7 & 29 & 17 & 5 & 2.73 & \\
\hline Median Delay & 33 & 43 & 63 & 47 & 44 & 49 & 38 & 73 & 48.8 & \\
\hline Total Errors & 31 & 82 & 67 & 53 & 11 & 36 & 80 & 67 & 3.34 & \\
\hline
\end{tabular}

1 The summary statistics are: (1) for frequency of $R P$, the response uncertainty, (2) for median delay, the mean of the median delay per $S P$, and $(3)$ for total errors, mean errors per $S$ per $S P$. 
sense the response uncertainty measures the size of the inferred subset of alternative psychological organizations of the sequence.

\section{Performance Measures}

Not only is the response uncertainty related to these physical properties of the sequence but also to the performance measures which are ordinarily considered to reflect the perceptual difficulty of the stimuli: the delay and errors to successful reproduction.

Delay. The delay score for each SP of a pattern presented in Table 1 is the median of the combined scores of all Ss who received that pattern or its complement. The use of the median avoided heavy weighting of occasional extreme delays in some cases by a single individual. The mean of these medians for all SPs is a summary statistic for each pattern.

The most quickly reproducible patterns, as shown by the average delays, are expansions, by repetition, of patterns of shorter unit-length, i.e., 2, or 4 . Thus this objective measure of uncertainty does correlate with performance. The rank-order correlation of the average delays for all patterns with the response uncertainty of the pattern is .88 . As the number of alternative ways of organizing the sequence increases, or in other words, as its complexity increases, so does the length of time required to listen, to perceive and to reproduce the pattern accurately to a criterion.

The delay measures the time required for $S$ to organize the pattern perceptually so that he can reproduce it. The simple patterns a re organized quickly and the complex ones more slowly. This difficulty of organization is particularly apparent in pattern $S$, which has an internal complementary symmetry which is retained no matter what the SP may be. This property produces a highly ambiguous pattern analogous to Rubin's figure-ground reversal patterns or Kopfermann's cubes on the one hand and to "hidden figures" on the other.

Errors. The total number of responses made after the initial delay and before reaching criterion and which were wrong are presented in Table 1 for each SP of the pattern. The summary statistic is the mean errors per S per SP in order to equate for differences in the number of different Ss and SPs for some of the patterns.

These errors differ considerably from pattern to pattern. They are correlated with both the response uncertainty and the delay. The mean errors for the patterns are rank-correlated .87 with delay and .86 with response uncertainty. Thus, another standard measure of the difficulty of a task is related to the number of alternative psychological organizations of a pattern, or to its complexity.

\section{Effect of SP}

Point of Response. If the SP of the sequence completely determined the RP, (i.e., the RP is at the same point as SP) the response uncertainty would be maximum and the contingent uncertainty (i.e., in- formation transmission) between SP and RP would also be maximum. In such a circumstance we would have no evidence of perceptual reorganization. This eventuality was not approached; the pattern having the greatest response uncertainty, pattern $T$, had a contingent uncertainty nearly as small as patterns $G$ and $\mathrm{K}$. While there was some small variability in the range of the contingent uncertainty among the patterns, the average contingent uncertainty was only 0.36 bits for patterns of 8 SPs and RPs, making it quite clear that the starting point of the pattern has little effect on the choice of the RP. In fact, this average value of the contingent uncertainty is not much greater than what would be obtained on a chance basis.

Performance Measures. These results have shown that where the sequence is started does not effect where $S$ starts responding in the sequence, i.e., does not affect how S perceptually organizes it. Nevertheless, it is possible that starting a sequence at a point which is consonant with S's ultimate organization could facilitate S's performance. Thus, measures of delay and errors should be lower for SPs which coincide with modal RPs.

Table 1 shows for each SP of each pattern the median delay and the total errors made regardless of the ultimate RP used by $\mathrm{S}$. The expectation of more efficient reorganization is confirmed for both measures. The mean delay for the two modal SPs in each pattern is 29.1 as compared to 33.9 for all other points. The mean errors for the two modal SPs are 19.4 as compared to 24.0 for all other points.

This effect is far more striking for the less complex patterns. For example, patterns E, F, G, and $H$ have a mean delay of 15 for the two modal points compared to $\mathbf{2 2 . 1}$ for all other points, and errors are similarly more differentiated. This fact itself is reasonable when we consider that the complex patterns have such long delays that any initial effect of SP must be dissipated by the time $S$ begins responding.

\section{DISCUSSION}

Phenomenologically, when one of these patterns is heard initially, it appears simply as a rather rapid sequence of individual elements. With continued listening, however, the series of elements becomes an organized entity, truly a pattern, and the simpler the pattern, the more rapidly this happens. So definite is its perception as a pattern with a beginning and an end that when a particular sequence terminates at other than its natural termination, the listener is left with a strong feeling of lack of completion.

After the perceptual organization has occurred, the pattern can be responded to in complete synchrony with little difficulty. Before that time, however, response to elements occurring at this rate is almost impossible with any degree of accurracy. In fact, our use of the delayed response technique as the primary experimental procedure was as much a matter of necessity 
as a matter of choice. Forced responding too early, particularly with the more difficult sequences, leads to almost complete disorganization, and actually interferes with the perceptual organization process.

Although these patterns are not perceived as a series of individual dichotomous elements, nevertheless the stimuli are just that, and so are not fundamentally unlike the stimuli used in so many sequential learning experiments. Most such experiments, however, involve probabilistically generated sequences, so that no pattern can occur, and comparison with our results is meaningless. Galanter and Smith (1958) used fixed and repeated sequences, but used too few and simple sequences to provide a basis of comparison with the more complicated patterns we have used. Restle (in press) reports some experiments and hypotheses of his own and of Keller from an unpublished dissertation which allow some degree of comparison of results, and which might clarify whether these more rapid auditory sequences lead to a qualitatively different kind of experience than the slowly presented sequences used in learning experiments.

Pattern difficulty. According to Restle, Keller's work showed that difficulty of learning sequences of different length was not related to length per se, but rather to the number of runs involved in the sequence before it repeated itself. Thus sequences of different length but with the same number of runs were equally difficult to learn.

The difficulty of perceptual organization of our patterns clearly has some relevance to the number of runs, although it is equally clear that more than that factor is involved. Patterns $E$ through $H$ have two runs each; patterns $J$ through $Q$ have four runs; and patterns $\mathbf{R}, \mathrm{S}$, and $\mathrm{T}$ have six runs. However, pattern $\mathrm{I}$, which also has six runs, is comparable in difficulty to patterns with just two runs. Furthermore, while the three most difficult patterns all have six runs, there are very great differences in the difficulties of these three patterns. Still further, the patterns with four runs have obvious differences in difficulty which are related to the length of the longest run (long runs being easier).

So patterns which are difficult to learn will, on the average, also be difficult to organize perceptually, but additional factors are involved in the perceptual organization process. As one example, our data make clear that a series of two or more single alternatives allows the pair of alternating elements to be perceived as a unit. In fact, it is probably this factor which makes pattern I, with its sequence of three single alternations so easy to organize.

Pattern organization. Restle presents data of his own concerned with the location of errors in learning of sequences nine units long. He shows that errors are low when the element in a sequence can be pre- dicted perfectly from runs of differing length (what he calls mandatory first-order rules); and that errors will be high when the element can not be predicted perfectly from runs of differing length. To illustrate, if 11 is always followed by 1 (i.e., the sequence 110 never occurs), then few errors will be made to the third 1. On the other hand, if both 110 and 111 occur in the sequence, then an optional rule is operating for determining the element after 11, and errors will be high. Since the existence of a large number of optional rules implies many runs in a sequence, these rules are in agreement with Keller's work that sequences with high numbers of runs are more difficult.

Our concern here, however, is with the location of salient points in the sequence. We have too few errors to allow meaningful analysis of errors at each possible position in the sequence. But one might expect that elements leading to low error would correspond to elements frequently used as RPs in our data, since points with low errors are those presumably perceived with high accuracy.

In this case there is no question that errors in Restle's sequences have little relevance to preferred RPs in ours. One can describe the location of our obtained preferred points in terms of mandatory first-order rules, but the generality is so limited that it seems unlikely that the nature of the mandatory rule is really the critical factor.

To illustrate, in the simple sequences, the preferred organization always starts with a mandatory reversal of the element, but such sequences should have essentially the same errors at all points, since all elements are completely predictable with first-order rules. Thus if errors should be related to preferred RPs, there should be no such preferences in these patterns. The converse, of course, is true, in that the simple patterns are the ones with the least response uncertainty; i.e., the strongest preferences.

Still further, with patterns having four runs, the most preferred RP is always one generated by a mandatory first-order rule following a run of two or more and calling for a reversal. But this rule itself is not consistent, and furthermore, mandatory first-order rules involving single alternations do not predict preferred RPs. To illustrate, pattern $K$ is organized primarily as 00101111, and this preferred starting point does occur mandatorily after the run 1111. But the secondary organization is 11110010 , and in this case the starting 1 is an optional prediction after the run of 0 . In addition, there exists in this sequence another organization, 10111100 , in which the starting point is a mandatory prediction after the run 00 , but this is a weakly preferred organization.

There are many other examples which make clear that these rules, which predict error locations, do not 
predict preferred organization of these patterns as determined by RP frequencies. While these cannot all be described, pattern $S$ does give an interesting contrast. The preferred organization is 10110100, and in this case the starting 1 is a mandatory prediction after a run of 00 . But this pattern organized as 01001011 accomplishes the same mandatory prediction for its starting point, yet is rarely used. On the other hand, 11010010 starts with an optionally predicted element, but is the second preferred organization.

The evidence is not totally convincing because of lack of enough direct contrasts of performance and types of sequence, but these comparisons certainly suggest that different psychological factors are involved in learning sequences at slow rates and in perceiving them at fast rates.

\section{References}

Day, R. H. Perception, Ann. Rev. Psychol., 1964, 15, 1-28. Farnsworth, P. R. The social psychology of music. New York: Dryden Press, 1958
Galanter, E. H., \& Smith, W. A. S. Some experiments on a simple thought-problem. Amer. J. Psy chol., 1958, 71, 359-366.

Garner, W. R. Uncertainty and structure as psychological concepts. New York: Wiley, 1962.

Garner, W. R., \& Clement, D. E. Goodness of pattern and pattern uncertainty. J. verbal Learn. verbal Behav., 1963, 2, 446-452.

Meyer, L. B. Emotion and meaning in music. Chicago: University of Chicago Press, 1956.

Restle, F. Grammatical analysis of the prediction of binary events. J. verbal Learn. verbal Behav., 1966, in press.

Woodrow, H. A quantitative study of thythm. Arch. Psychol., 1909, $14,1-66$.

\section{Hotes}

1. The authors wish to acknowledge the kind assistance of Commander A. D. Garvin and Mr. Dale Meffley of the Service School Command, U. S. Naval Training Center, Bainbridge, Maryland, in providing the subjects and space for conducting this study:

2. Now at Veterans Administration Hospital, Brecksville, Ohio. 3. Pattern A (11111111 and its complement) has been omitted from Table 1. Although in fact the sequences were used, they are relatively meaningless; there is no possibility for an error score, there is no possibility of measuring the uncertainty of the point of response and the delay (17.) measured only the length of time before the $\mathbf{S}$ was confident that no other stimulus would occur. 